\title{
Relationship between Marital Status and Metabolic Syndrome in Korean Middle- Aged Women: The Sixth Korea National Health and Nutrition Examination Survey (2013-2014)
}

\author{
Young-Ah Jung, Li-Ly Kang, Ha-Nui Kim, Hoon-Ki Park*, Hwan-Sik Hwang, Kye-Yeung Park \\ Department of Family Medicine, Hanyang University College of Medicine, Seoul, Korea
}

\begin{abstract}
Background: This study aimed to investigate the relationship between marital status and the incidence of metabolic syndrome in Korean middle-aged women.

Methods: Based on data from the sixth Korea National Health and Nutrition Examination Survey (2013-2014), 3,225 women aged 40-69 years were subjected to the analysis. Marital status was categorized as married, unmarried, separated, widowed, or divorced. The odds ratios (ORs) for metabolic syndrome were calculated based on marital status. After adjustment for age, income level, education level, alcohol intake, smoking status, leisure physical activity, menopause status, daily calories, and fat intake, changes in the OR for metabolic syndrome based on marital status were examined by multivariate logistic regression analysis.

Results: The OR for metabolic syndrome in the widowed group to the married group was 4.818 (95\% confidence interval [CI], 3.861-6.002; $\mathrm{P}<0.001$ ) and that after adjustment of age, economic level, education level, alcohol intake, smoking status, physical activity, menopause status, total daily calories, and fat intake was 2.141 (CI, 1.432-3.199; $\mathrm{P}<0.001$ ), both of which were statistically significant. The $\mathrm{OR}$ for metabolic syndrome in the unmarried group to the married group was 0.246 (CI, 0.141-0.431; $\mathrm{P}<0.001$ ) after adjustment of all components. On the contrary, the ORs of the separated group and the divorced group to the married group were not significant.

Conclusion: In comparison with the married middle-aged group, the widowed middle-aged group tended to have a higher risk of metabolic syndrome, which is speculated to be related to socioeconomic factors and health behavior.
\end{abstract}

Keywords: Metabolic Syndrome; Marital Status; Middle Aged; Women; Health Behavior

Received: February 24, 2017, Revised: August 31, 2017, Accepted: August 31, 2017

*Corresponding Author: Hoon-Ki Park https://orcid.org/0000-0002-8242-0943

Tel: +82-2-2290-8740, Fax: +82-2-2281-7279, E-mail: Hoonkp@hanyang.ac.kr 


\section{INTRODUCTION}

To date, studies showed that those who were married and lived with a spouse had better health behavior than those who lived without a spouse. ${ }^{1)}$ Changes in marital status, such as separation or widowhood, tend to adversely affect health; particularly, married men had a lower mortality rate owing to cardiovascular diseases than men who were unmarried, separated, divorced, or widowed. ${ }^{2)}$ In a study (the Million Women Study) of 734,626 English women aged 55-64 years conducted over 8.8 years, women with a spouse had a lower risk of mortality owing to ischemic heart disease. ${ }^{3)}$ Data from the Korea National Health and Nutrition Examination Survey (KNHANES) from 2007 to 2010 showed that marital status was associated with health conditions, and that there were gender-based differences in health behaviors. ${ }^{4,5)}$ In the Healthy Women Study conducted in Pittsburg that followed up 413 women aged $42-50$ years over 11.5 years, the prevalence of metabolic syndrome was higher in women who were not satisfied with their married life or were widowed than in those who were satisfied with married life. ${ }^{6)}$

The risk factors for metabolic syndrome, including abdominal obesity, hypertriglyceridemia, low high-density lipoprotein cholesterol levels, hyperglycemia, and high blood pressure, were found to be closely related with the risk factors for cardiovascular disease and type 2 diabetes. ${ }^{6}$ In particular, the prevalence of metabolic syndrome in Korean women tended to increase with increasing age and the prevalence of metabolic syndrome in women aged 50 years or older tended be over two-folds higher than that in men. ${ }^{7)}$ However, no study was conducted to examine the relationship of marital status with these chronic diseases and health in Korea, particularly among middle-aged women. This study aimed to investigate the relationship between marital status and the prevalence of metabolic syndrome in Korean middle-aged women.

\section{METHODS}

\section{Respondents}

The KNHANES identified the health level, health-related recognition and behavior, and the food and nutrition intake of people. In this study, data from the KNHANES from 2013 to 2014 were obtained. Among 15,568 persons who participated in the sixth KNHANES, we selected 3,625 women aged 40-69 years. We selected 3,225 women who completed all three components (the Health Interview Survey, the Nutrition Survey, and the Health Examination Study) of the KNHANES. This cross-sectional study was conducted in 3,225 women aged $40-69$ years.

\section{Measurement of Variables}

\section{1) Marital status}

Marital status was categorized into five groups. If the respondent answered "No" to the question "Have you ever been married?", the person was regarded as unmarried, the person was regarded as unmar- ried, whereas those who answered "yes" was asked to answer the second question. As regards questions on current marital status, those who answered "had spouse and were living together" were included in the married group; those who answered "had spouse, but did not live together" were included in the separated group; those who answered "had no spouse due to death of spouse" were included in the widowed group; and those who answered "had no spouse due to divorce" were included in the divorced group.

\section{2) Data collection and measurement of covariates}

The indicators of socioeconomic status applied were income level and education level. For income level, respondents who belonged to the lowest quartile were regarded as the low-income group, those who belonged to the mid-low and mid-high income quartiles were regarded as the medium-income group, and those who belonged to the highest quartile were regarded as the high-income group. Education level was divided into three groups-middle school graduate or lower, high school graduate, and college graduate or higher.

Indices for health behavior were based on the data from the health survey and nutrition examination survey. In questions on current smoking status, the smoking group and occasional smoking group were considered current smokers, and those who answered that they had smoked previously were considered former smokers. The nonsmoking group and not applicable group were considered non-smokers.

According to the criteria of the US National Institute on Alcohol Abuse and Alcoholism, the standard drinking volume is $14 \mathrm{~g}$. In questions about alcohol consumption per episode, the respondents were classified into the following groups: individuals who consumed $<3$ cups (less than $14 \mathrm{~g}$ ) per episode, individuals who consumed 3 to $\leq 7$ cups $(14 \mathrm{~g}$ to $<40 \mathrm{~g}$ ) per episode, or individuals who consumed $>7$ cups (>40 g) per episode. ${ }^{\text {8) }}$

In questions on physical activity performed during leisure time in a week, the respondents were divided into groups with muscle strengthening exercise or flexibility exercise performed at least 4 times or with exercise performed three times or fewer.

In questions about menopause, the respondents were classified into premenopausal group and postmenopausal groups.

Dietary intake was assessed using the single 24-hour dietary recall method. Experienced interviewers instructed the respondents to recall and describe the foods consumed over the previous 24 hours. Food models and measuring bowls, cups, and spoons were used to assist in estimating portion sizes. The record for each respondent was coded, and standard reference tables were used to convert weights to grams.

The body mass index (BMI) value was calculated by dividing body weight in kilograms by height in meters squared. Waist circumference was measured at a level between the lower rib margin and the top of the iliac crest. The blood pressure in the upper arm of resting respondents was measured using an automatic blood pressure measuring device after stabilizing the respondents for more than 10 minutes. Blood was collected after fasting for more than 8 hours. 
National Cholesterol Education Program's Adult Treatment Panel III (NCEP-ATP III) criteria were followed for the diagnosis of metabolic syndrome. ${ }^{9)}$ The waist circumference used was $\geq 80 \mathrm{~cm}$, which is the standard measurement for Korean women. Metabolic syndrome was diagnosed when at least three of the following five items were satisfied: (1) waist circumference: $\geq 80 \mathrm{~cm}$; (2) triglyceride: $\geq 150 \mathrm{mg} / \mathrm{dL}$; (3) high-density lipoprotein cholesterol: $\leq 50 \mathrm{mg} / \mathrm{dL}$; (4) blood pressure: $\geq 130 / 85 \mathrm{~mm}$ Hg or taking a hypotensive agent; and (5) fasting glucose: $\geq 100 \mathrm{mg} / \mathrm{dL}$ or previously diagnosed with type II diabetes.

\section{3) Statistical analysis}

To reflect the stratified cluster and systematic sampling and non-responders in the KNHANES, test and nutrition examination survey data were weighted for the analysis. Socioeconomic factors, BMI, and health behavior were compared. The general linear model was used for continuous variables, while the independence test was used for categorical variables.

Multivariate logistic regression analysis was conducted with marital status as independent variable and diagnosis with metabolic syndrome as the dependent variable. The odds ratios for metabolic syndrome were calculated depending on unmarried, separated, widowed, and divorced statuses. Changes in odds ratio for metabolic syndrome in each marital status were examined after adjustment for age, income level, education level, alcohol intake, smoking status, leisure time physical activity, menopause status, daily calories, and fat intake.

The statistical program used was IBM SPSS ver. 21.0 (IBM Corp., Armonk, NY, USA), and P-value $<0.05$ was considered statistically significant.

\section{RESULTS}

\section{General Characteristics of Respondents}

There were 3,225 respondents including 2,648 women in the married group (83.7\%), 54 in the unmarried group (1.5\%), 26 in the separated group $(0.8 \%), 308$ in the widowed group (8.2\%), and 189 in the divorced group (5.9\%) (Table 1). The respondents in the widowed group and the unmarried group had an average age of 60.3 and 48.8 years, respectively, were identified as the oldest and youngest groups, respectively. The widowed group had the largest waist circumference $(81.92 \mathrm{~cm})$. The unmarried group had the highest level of education; $39.9 \%$ of them were college graduates or achieved a higher level of education. The married group had the highest income, with $28.45 \%$ of them in the top three quartiles. The unmarried group had a significantly higher alcohol intake (21.0\%) and had the highest proportion of smokers (23.4\%). The widowed group had the highest proportion of postmenopausal women (41\%) and had the lowest daily calorie and

Table 1. Characteristics of the 3,225 study participants according to marital status

\begin{tabular}{|c|c|c|c|c|c|c|}
\hline Characteristic & Married $(n=2,648)$ & Unmarried $(n=54)$ & Separated $(n=26)$ & Widowed $(n=308)$ & Divorced $(n=189)$ & P-value \\
\hline $\operatorname{Age}(y)^{*}$ & $51.85 \pm 0.19$ & $48.76 \pm 0.85$ & $53.14 \pm 1.3$ & $60.25 \pm 0.48$ & $52.27 \pm 0.64$ & $<0.001$ \\
\hline Body mass index $\left(\mathrm{kg} / \mathrm{m}^{2}\right)^{\star}$ & $23.69 \pm 0.08$ & $23.83 \pm 0.66$ & $23.70 \pm 0.68$ & $24.26 \pm 0.24$ & $23.27 \pm 0.25$ & 0.08 \\
\hline Waist circumference $(\mathrm{cm})^{*}$ & $78.40 \pm 0.23$ & $78.85 \pm 1.61$ & $78.40 \pm 2.18$ & $81.92 \pm 0.63$ & $78.11 \pm 0.68$ & 0.05 \\
\hline Education $^{\dagger}$ & & & & & & $<0.001$ \\
\hline Middle school & $35.9(1,061)$ & $17.5(11)$ & $50.1(17)$ & $77.2(244)$ & $39.5(84)$ & \\
\hline High school & $40.9(1,013)$ & $42.6(25)$ & $37.0(6)$ & $18.3(48)$ & $45.0(80)$ & \\
\hline College & $23.2(580)$ & $39.9(22)$ & $12.9(3)$ & $4.5(16)$ & $15.5(29)$ & \\
\hline Income $^{\dagger}$ & & & & & & $<0.001$ \\
\hline Low & $44.4(1,280)$ & $93.9(54)$ & $69.3(20)$ & $65.4(209)$ & $82.3(172)$ & \\
\hline Middle & $27.2(782)$ & $0.8(2)$ & $24.3(6)$ & $18.2(61)$ & $10.1(23)$ & \\
\hline High & $28.4(796)$ & $5.3(4)$ & $6.2(2)$ & $16.3(52)$ & $7.6(17)$ & \\
\hline Alcohol intake $(\mathrm{g})^{\dagger}$ & & & & & & $<0.001$ \\
\hline$<14$ & $57.2(1,509)$ & $34.9(18)$ & $49.2(13)$ & $73.6(224)$ & $45.8(85)$ & \\
\hline$\leq 14$ or $<40$ & $35.2(926)$ & $44.2(24)$ & $44.2(11)$ & $23.8(71)$ & $36.0(68)$ & \\
\hline$\geq 40$ & $7.6(185)$ & $21.0(11)$ & $6.6(2)$ & $3.2(9)$ & $18.2(34)$ & \\
\hline Smoking $^{\dagger}$ & & & & & & $<0.001$ \\
\hline Never & $94.2(2,555)$ & $73.3(45)$ & $73.8(20)$ & $92.2(286)$ & 70.6 (144) & \\
\hline Former & $2.7(71)$ & $9.5(3)$ & $2.8(1)$ & $2.5(9)$ & $12.9(21)$ & \\
\hline Current & $3.1(78)$ & $17.2(10)$ & $23.4(5)$ & $5.3(18)$ & $16.4(34)$ & \\
\hline Physical activity $(\mathrm{d} / \mathrm{wk})^{\dagger}$ & & & & & & 0.192 \\
\hline$<3$ & $58.9(1,566)$ & $68.8(39)$ & $51.1(14)$ & $65.4(202)$ & $56.9(106)$ & \\
\hline$\geq 3$ & $41.1(1,087)$ & $31.2(18)$ & $48.9(12)$ & $34.6(107)$ & $43.1(87)$ & \\
\hline Menopause status $^{\dagger}$ & & & & & & $<0.001$ \\
\hline Premenopausal women & $78.3(2,065)$ & $99.5(53)$ & $69.4(17)$ & $58.1(178)$ & $73.7(138)$ & \\
\hline Postmenopausal women & $21.7(553)$ & $0.5(2)$ & $30.6(7)$ & $41.9(126)$ & $26.3(49)$ & \\
\hline Daily calories $(\mathrm{kcal} / \mathrm{d})^{*}$ & $2,092.15 \pm 14.08$ & $2,073.53 \pm 21.46$ & $1,942.28 \pm 167.08$ & $1,533.15 \pm 23.78$ & $1,942.56 \pm 59.91$ & $<0.001$ \\
\hline Fat $(g / d)^{*}$ & $44.71 \pm 0.55$ & $55.55 \pm 0.86$ & $38.06 \pm 4.69$ & $22.45 \pm 0.73$ & $39.63 \pm 2.13$ & $<0.001$ \\
\hline
\end{tabular}


fat intake.

\section{Prevalence of Metabolic Syndrome Based on Marital Status}

The prevalence of metabolic syndrome was $30 \%$ in the married group, $34.2 \%$ in the unmarried group, $33.5 \%$ in the separated group, $58.2 \%$ in the widowed group, and $35.8 \%$ in the divorced group (Table 2). The prevalence of each of five components for metabolic syndrome had a significant difference based on the marital status $(\mathrm{P}<0.001)$.

\section{Odds Ratio for Metabolic Syndrome Based on Marital Status}

The unadjusted odds ratio of the widowed group to the married group was 4.814 (confidence interval [CI], 3.861-6.002; $\mathrm{P}<0.001$ ) (Table 3). When only age and socioeconomic factors (household income and education level) were adjusted, the odds ratio was 2.341 (CI, 1.7973.148; $\mathrm{P}<0.001$ ), and when behavioral risk factors (alcohol consumption frequency, smoking status, physical activity, menopausal status, total daily calories, and fat intake) were additionally adjusted, the odds ratio was 2.141 (CI, 1.432-3.199; $\mathrm{P}<0.001$ ). Moreover, the odds ratio for metabolic syndrome in the unmarried group to the married group was 0.246 (CI, 0.141-0.431; $\mathrm{P}<0.001$ ) after adjustment of all components. However, the odds ratios of the separated group, and divorced group to married group were not considered significant.

\section{DISCUSSION}

In this study, the unadjusted odds ratio for metabolic syndrome of the widowed group to the married group was 4.814 (CI, 3.861-6.002; $\mathrm{P}<0.001)$. The odds ratio for metabolic syndrome in the widowed group to the married group was 2.341 (CI, 1.797-3.148; $\mathrm{P}<0.001$ ) after adjustment of age and socioeconomic factors only, and 2.141(CI, 1.432-3.199; $\mathrm{P}<0.001$ ) upon additional adjustment for health behavior. The odds ratio for metabolic syndrome in the unmarried group to the married group was 0.246 (CI, 0.141-0.431; $\mathrm{P}<0.001$ ) after adjustment of all components. These results may indicate that age, socioeconomic factors, and health behavior based on marital status affected the incidence of metabolic syndrome.

We identified that many factors affect the prevalence of metabolic syndrome in this study. We identified that the widowed group had a significantly larger waist circumference (Table 1), suggesting that abdominal obesity has an effect as one of the NCEP-ATP III criteria for the diagnosis of metabolic syndrome. Jouyandeh et al. ${ }^{10)}$ reported that there was a significant association between the number of metabolic syndrome components and increasing waist circumference. A previous study showed an increased risk of metabolic syndrome in postmenopausal women especially in developing countries and Asia. ${ }^{11)}$

Many previous studies have reported that a low socioeconomic status was associated with high mortality rate owing to the development of cardiovascular diseases. ${ }^{12-15)}$ In this study, the widowed group had lower household income and lower education level than the married group. Consistently, a study has reported that Korean adults who were unemployed or with low education levels had a higher prevalence of metabolic syndrome. ${ }^{7)}$

Compared to the married group, the widowed group had a higher proportion of smokers. It has been speculated that divorced, separat-

Table 2. Prevalence of metabolic syndrome according to marital status in Korean middle-aged women

\begin{tabular}{lccccrr}
\hline \multicolumn{1}{c}{ Variable } & \multicolumn{1}{c}{ Married } & \multicolumn{1}{c}{ Unmarried } & \multicolumn{1}{c}{ Separated } & Widowed & Divorced & P-value \\
\hline Large WC & $40.2(37.9-42.5)$ & $41.0(29.0-54.3)$ & $37.8(20.6-58.8)$ & $57.3(50.8-63.4)$ & $38.8(31.8-46.3)$ & $<0.001$ \\
High TG* & $31.0(29.1-33.0)$ & $40.0(26.2-55.5)$ & $45.1(24.1-68.0)$ & $45.9(39.4-52.6)$ & $39.3(32.3-46.8)$ & 0.015 \\
Low HDL-C* & $41.2(39.1-43.4)$ & $50.9(35.9-65.9)$ & $53.0(29.6-75.2)$ & $59.4(53.0-65.4)$ & $44.0(36.7-51.5)$ & 0.008 \\
High BP & $35.5(33.4-37.4)$ & $25.9(16.4-38.3)$ & $30.6(13.7-55.0)$ & $56.2(50.1-62.1)$ & $37.6(30.1-45.8)$ & $<0.001$ \\
High FBS & $16.3(14.7-18.0)$ & $12.6(6.0-24.6)$ & $22.8(9.6-45.1)$ & $27.8(21.9-34.7)$ & $18.6(12.7-26.5)$ & 0.003 \\
Metabolic syndrome & $30.0(28.0-32.1)$ & $34.2(21.1-50.3)$ & $33.5(16.2-56.8)$ & $58.2(51.3-64.7)$ & $35.8(28.4-44.0)$ & $<0.001$ \\
\hline
\end{tabular}

Values are presented as \% (range). Each component was determined by analysis of variance: large WC $\geq 85 \mathrm{~cm}$; high TG $\geq 150 \mathrm{mg} / \mathrm{dL}$; low HDL-C $<50 \mathrm{mg} / \mathrm{dL}$ for women; high $\mathrm{BP} \geq 130 / 85 \mathrm{~mm} \mathrm{Hg}$; and high FBS $\geq 100 \mathrm{mg} / \mathrm{dL}$.

WC, waist circumference; TG, triglyceride; HDL-C, high-density lipoprotein cholesterol; BP, blood pressure; FBS, fasting blood sugar.

*Using log-transformed data.

Table 3. ORs of metabolic syndrome in Korean middle-aged women according to marital status

\begin{tabular}{|c|c|c|c|c|c|c|}
\hline Marital status & Unadjusted OR (95\% Cl) & P-value & Adjusted OR* $(95 \% \mathrm{Cl})$ & P-value & Adjusted OR ${ }^{\dagger}(95 \% \mathrm{Cl})$ & P-value \\
\hline Married & 1 & & 1 & & 1 & \\
\hline Unmarried & $0.075(0.037-0.151)$ & 0.568 & $0.045(0.029-0.069)$ & $<0.001$ & $0.246(0.141-0.431)$ & $<0.001$ \\
\hline Separated & $1.238(0.436-3.518)$ & 0.738 & $1.013(0.373-2.750)$ & 0.352 & $0.790(0.202-3.087)$ & 0.516 \\
\hline Widowed & 4.814 (3.861-6.002) & $<0.001$ & 2.341 (1.797-3.148) & $<0.001$ & 2.141 (1.432-3.199) & $<0.001$ \\
\hline Divorced & $1.375(0.907-2.084)$ & 0.138 & $1.174(0.838-1.644)$ & 0.187 & $0.814(0.498-1.330)$ & 0.352 \\
\hline
\end{tabular}

Analyzed using multivariate logistic regression.

$\mathrm{OR}$, odds ratio; $\mathrm{Cl}$, confidence interval.

${ }^{*}$ Adjusted for age, education, and income. ${ }^{\dagger}$ Adjusted for age, education, income, alcohol intake, smoking, physical activity, menopause status, and daily calorie and fat intake. 
ed, or widowed women experienced stress owing to financial burdens and the responsibility of raising children, resulting in an increase in the proportion of smokers. Smoking interferes with insulin function, leading to insulin resistance, ${ }^{16)}$ and causes abdominal obesity, ${ }^{17)}$ elevation of blood pressure through activation of the autonomic nervous system and increase in myocardial oxygen demand, ${ }^{18)}$ increase in blood triglyceride levels, and reduction of high-density lipoprotein cholesterol. $^{19)}$

Moderate alcohol consumption ( $<15 \mathrm{~g} / \mathrm{d}$ ) has a positive effect on the elevation of high-density lipoprotein levels, which was also consistent with the observation in this study as evidenced by the higher rate of moderate alcohol consumption in the married group; therefore, moderate alcohol consumption can lead to the occurrence of metabolic syndrome. However, data on heavy alcohol consumption were not included in this study, and it was difficult to identify the relationship between metabolic syndrome and alcohol consumption. ${ }^{20)}$

Physical activity during leisure time among women aged 40 years or older is related to the incidence of metabolic syndrome. ${ }^{21)}$ This study failed to identify the significant relationship between physical activity and metabolic syndrome, which seems to be attributable to a slight modification by error in the questionnaire survey.

Troxel et al. ${ }^{6)}$ have reported that women who were not satisfied with their married life had a higher prevalence of metabolic syndrome than those who were satisfied with their married life or were widowed, suggesting that the presence of a spouse or a positive relationship provides physical and psychological support, especially providing a buffer in stressful situations. In addition, it was indicated that married people are healthier because they experience lesser stress and because of the presence of their spouse, which promote a healthy lifestyle. ${ }^{22)}$

These findings are consistent with the results of a previous study, reporting that there were differences in socioeconomic factors and risky health behavior based on marital status, and these differences should affect the prevalence of metabolic syndrome.

This study had some limitations. It was difficult to explain the temporal or causal relationships between marital status and metabolic syndrome owing to the cross-sectional nature of this study. Nevertheless, since these data were obtained after adjustment of various socioeconomic factors and the risk factors of behavior affecting metabolic syndrome, the relationships may be meaningful. In addition, the health behaviors were investigated not on the basis of objective measurement but on the subjective responses of the participants; therefore, a recall bias may occur and the measurement could be affected by other confounding factors in addition to the variables considered in this study. Furthermore, because it was impossible to identify the effects by time points of change in marital status, it was also impossible to identify the socioeconomic factors and health behaviors at the times of change.

In this study, on adjustment for age, socioeconomic factors, and health behavior risk factors, middle-aged widowed women had a significantly higher risk for metabolic syndrome compared than married women, which suggests that socioeconomic factors and health behav- iors of middle-aged widowed women are associated with the prevalence of metabolic syndrome. In some studies, divorced men were likely to have metabolic syndrome than married men, and this finding may be mediated by their risky health behavior. ${ }^{23)}$ The strength of this paper is that a high prevalence of metabolic syndrome was found among widowed women in Korea, which is associated with health behavior. Therefore, we could speculate that widowed women have the possibility of developing metabolic syndrome. Because marital status appears to be related to cardiovascular disease in Korean middle-aged women, it is important to provide lipid screening and educational programs to improve women's lifestyle based on their marital status. To explain the temporal or causal relationships between marital status and metabolic syndrome, prospective studies should be conducted in the future.

\section{CONFLICT OF INTEREST}

No potential conflict of interest relevant to this article was reported.

\section{REFERENCES}

1. Schoenborn CA. Marital status and health: United States, 1999-2002. Adv Data 2004;(351):1-32.

2. Ben-Shlomo Y, Smith GD, Shipley M, Marmot MG. Magnitude and causes of mortality differences between married and unmarried men. J Epidemiol Community Health 1993;47:200-5.

3. Floud S, Balkwill A, Canoy D, Wright FL, Reeves GK, Green J, et al. Marital status and ischemic heart disease incidence and mortality in women: a large prospective study. BMC Med 2014;12:42.

4. Yim HJ, Park HA, Kang JH, Kim KW, Cho YG, Hur YI, et al. Marital status and health behavior in middle-aged Korean adults. Korean J Fam Med 2012;33:390-7.

5. Yim HJ, Park HA, Kang JH, Kim KW, Cho YG, Hur YI, et al. Marital status and health behaviors in middle-aged Korean adults: from the 2007-2010 Korea National Health and Nutrition Examination Survey. Korean J Health Promot 2012;12:178-86.

6. Troxel WM, Matthews KA, Gallo LC, Kuller LH. Marital quality and occurrence of the metabolic syndrome in women. Arch Intern Med 2005;165:1022-7.

7. Park HS, Oh SW, Cho SI, Choi WH, Kim YS. The metabolic syndrome and associated lifestyle factors among South Korean adults. Int J Epidemiol 2004;33:328-36.

8. National Institute on Alcohol Abuse and Alcoholism. Helping patients who drink too much: a clinician's guide: updated 2005 edition [Internet]. Bethesda (MD): National Institutes of Health; 2005 [cited 2014 Mar 13]. Available from: https://www.integration.samhsa.gov/clinical-practice/Helping_Patients_Who_Drink_Too_Much.pdf.

9. Expert Panel on Detection, Evaluation, and Treatment of High Blood Cholesterol in Adults. Executive summary of the third report of the National Cholesterol Education Program (NCEP) Expert Panel on Detection, Evaluation, and Treatment of High Blood Cholesterol in Adults (Adult Treatment Panel III). JAMA 2001;285:2486-97.

10. Jouyandeh Z, Nayebzadeh F, Qorbani M, Asadi M. Metabolic syn- 
drome and menopause. J Diabetes Metab Disord 2013;12:1. https:// doi.org/10.1186/2251-6581-12-1.

11. Meigs JB. Invited commentary: insulin resistance syndrome? Syndrome X? Multiple metabolic syndrome? A syndrome at all?: factor analysis reveals patterns in the fabric of correlated metabolic risk factors. Am J Epidemiol 2000;152:908-11.

12. Egbujie BA, Igumbor EU, Puoane T. A cross-sectional study of socioeconomic status and cardiovascular disease risk among participants in the Prospective Urban Rural Epidemiological (PURE) Study. S Afr Med J 2016;106:900-6.

13. Yu Z, Nissinen A, Vartiainen E, Song G, Guo Z, Zheng G, et al. Associations between socioeconomic status and cardiovascular risk factors in an urban population in China. Bull World Health Organ 2000;78:1296305 .

14. Kaplan GA, Keil JE. Socioeconomic factors and cardiovascular disease: a review of the literature. Circulation 1993;88(4 Pt 1):1973-98.

15. Clark AM, DesMeules M, Luo W, Duncan AS, Wielgosz A. Socioeconomic status and cardiovascular disease: risks and implications for care. Nat Rev Cardiol 2009;6:712-22.

16. Attvall S, Fowelin J, Lager I, von Schenck H, Smith U. Smoking induces insulin resistance: a potential link with the insulin resistance syn- drome. J Intern Med 1993;233:327-32.

17. Shimokata H, Muller DC, Andres R. Studies in the distribution of body fat: III. effects of cigarette smoking. JAMA 1989;261:1169-73.

18. Najem B, Houssiere A, Pathak A, Janssen C, Lemogoum D, Xhaet O, et al. Acute cardiovascular and sympathetic effects of nicotine replacement therapy. Hypertension 2006;47:1162-7.

19. Kong C, Nimmo L, Elatrozy T, Anyaoku V, Hughes C, Robinson S, et al. Smoking is associated with increased hepatic lipase activity, insulin resistance, dyslipidaemia and early atherosclerosis in Type 2 diabetes. Atherosclerosis 2001;156:373-8.

20. Bleich S, Bleich K. Moderate alcohol consumption and the risk of cardiovascular disease. Am J Clin Nutr 2002;75:948.

21. Park MY, Kim SH, Cho YJ, Chung RH, Lee KT. Association of leisure time physical activity and metabolic syndrome over 40 years. Korean J Fam Med 2014;35:65-73.

22. Wyke S, Ford G. Competing explanations for associations between marital status and health. Soc Sci Med 1992;34:523-32.

23. Chung TH, Kim MC, Choi CH, Kim CS. The association between marital status and metabolic syndrome in Korean men. Korean J Fam Med 2010;31:208-14 